\title{
Viability Discrimination of a Class of Control Systems on a Nonsmooth Region
}

\author{
Na Zhao, ${ }^{1}$ Jianfeng Lv, ${ }^{1}$ Jinlin Yang, ${ }^{1}$ and Xinzhi Liu ${ }^{2}$ \\ ${ }^{1}$ School of Mathematics, Physics and Biological Engineering, Inner Mongolia University of Science and Technology, \\ Baotou 014010, China \\ ${ }^{2}$ Department of Applied Mathematics, University of Waterloo, Waterloo, ON, Canada N2L 3G1
}

Correspondence should be addressed to Jianfeng Lv; lvjianfeng2006@126.com

Received 27 July 2013; Revised 16 December 2013; Accepted 18 December 2013; Published 12 January 2014

Academic Editor: Zhigang Jiang

Copyright (C) $2014 \mathrm{Na}$ Zhao et al. This is an open access article distributed under the Creative Commons Attribution License, which permits unrestricted use, distribution, and reproduction in any medium, provided the original work is properly cited.

\begin{abstract}
The viability problem is an important field of study in control theory; the corresponding research has profound significance in both theory and practice. In this paper, we consider the viability for both an affine nonlinear hybrid system and a hybrid differential inclusion on a region with subdifferentiable boundary. Based on the nonsmooth analysis theory, we obtain a method to verify the viability condition at a point, when the boundary function of the region is subdifferentiable and its subdifferential is convex hull of many finite points.
\end{abstract}

\section{Introduction}

Hybrid systems have been used to describe complex dynamic systems that involve both continuous and discrete systems. Such hybrid systems can be extensively used in robotics, automated highway systems, air traffic management systems, manufacturing, communication networks, and computer synchronization, and so forth. There has been significant research activity in the area of hybrid systems in the past decade involving researchers from several areas [1-8]. In recent years, the viability of systems is an important research topic; it has been widely used in both reach-ability and designing security domain.

In the study of hybrid systems, the concept of viability is more prevalent. The notion of viability was first introduced by Aubin [9]. Viability property provides a very nice theoretical framework for a hybrid controller design problem. Many researchers have considered the problem of viability for the analysis and control of hybrid systems [10-14]. The nonsampling viability problem was examined in the pioneering work of Aubin and coworkers [10] in which impulse differential inclusions are used to describe hybrid behavior.

As an important part of hybrid system, studies in the viability theory include two topics. One is to verify viability condition for a given set. Another one is to design a viable solution within a viable set. Viability conditions for a linear control system have been studied widely in recent years; see $[15,16]$. A necessary and sufficient viability condition for a differential inclusion was given in $[8,17]$, but it is a hard work to check that condition in most applications directly. In the literature [10], the authors give the necessary and sufficient condition of the viability, but it is still very difficult to judge quantitatively. Gao in [18] discusses the viability discrimination for an affine nonlinear control system on a smooth region; it gives some results on continuous system. There is certain limitation in the application of the literature [18]. The limitation is that the region must be smooth; in fact most of the region's boundaries are nonsmooth. Ahmed considers the viability criteria for a hybrid differential inclusions on smooth region in [19]. Gao in [20] gives viability criteria for differential inclusions on a nonsmooth region.

In this paper, we mainly consider the viability condition of a hybrid differential inclusion on a region with subdifferentiable boundary. Based on nonsmooth analysis theory, a method for checking the validity of the viability is given for such case as mapping of the set valued at the right hand of the differential inclusion is a polyhedron, the 
boundary function of the region is sub-differentiable, and its sub-differential is a convex hull with finite point set.

The paper is organized as follows. Section 2 states the main assumption, definitions and describes the hybrid dynamics. Section 3 overcomes these limitations in the literature [18]; we deal with the viability criteria for a hybrid system on a region with sub-differentiable boundary. Section 4 considers the viability of a hybrid differential inclusion. Section 5 shows an example.

\section{Preliminaries}

Consider the general form of nonlinear control system

$$
\dot{x}(t)=f(x, u), \quad u \in U,
$$

where $x \in \mathbb{R}^{n}$ denotes the state variable, $u \in U$ denotes the control variable, $U \subset \mathbb{R}^{m}$, and $f(x, u)$ is a Lipschitz function which is from $\mathbb{R}^{m+n}$ to $\mathbb{R}^{n}$.

Definition 1 (see [8]). Let $W \subset \mathbb{R}^{n}$ be a subset of $\mathbb{R}^{n}$, for any initial states $x_{0} \in W$, if there exists one solution $x(t)$ of the system (1), such that $x(t) \in W$ for all $t \geq 0$; then we call the subset $W$ viable under the system (1); the solution $x(t)$ is called viable solution.

Definition 2 (see [8]). Let $K \subseteq \mathbb{R}^{n}$ be a nonempty subset of $\mathbb{R}^{n}$; the tangent cone of the set $K$ at $x \in K$ is given by the formula

$$
T_{K}(x)=\left\{v \in \mathbb{R}^{n} \mid \liminf _{t \rightarrow 0^{+}} d_{K}(x+t v)=0\right\},
$$

where $d_{K}(y)$ is distance from the point $y \in \mathbb{R}^{n}$ to the set $K$.

Definition 3. Let $F(\cdot): X \rightarrow 2^{X}$ be a set valued map, it is said to be upper semicontinuous if for all $x^{0} \in X$ and each $\epsilon>0$, there exists $\delta>0$, such that $\left\|x-x^{0}\right\|<\delta$ implies $F(x) \subseteq F\left(x^{0}\right)+\epsilon B$ for all $x \in X$; that is, $F(x) \subseteq B\left(F\left(x^{0}\right), \epsilon\right)$.

Definition 4. Let $F(\cdot): X \rightarrow 2^{X}$ be a set valued map; $F$ is said to be Marchaud if the following conditions hold:

(i) $F$ is upper semicontinuous;

(ii) $F(x)$ is a nonempty convex compact set for all $x \in X$;

(iii) $F$ is linear growth; that is, there exists $\alpha>0$, such that

$$
\sup \{\|v\| \mid v \in F(x)\} \leq \alpha(\|x\|+1)
$$

for all $x \in X$.

Definition 5. Let $F(\cdot): X \rightarrow 2^{X}$ be a set valued map, if there exists a constant $\lambda>0$ such that

$$
F\left(x^{1}\right) \subseteq F\left(x^{2}\right)+\lambda\left\|x^{1}-x^{2}\right\| B(0,1)
$$

for all $x^{1}, x^{2} \in X$, then $F$ is said to be Lipschitz, where $\lambda>0$ is a Lipschitz constant.

Definition 6 (see [10], hybrid differential inclusion). A hybrid differential inclusion is a collection $H=(X, F, R, J)$, consisting of a finite dimensional vector space $X$, a set valued map
$F: X \rightarrow 2^{X}$, regarded as a differential inclusion $\dot{x}(t) \in F(x)$, a set valued map $R: X \rightarrow 2^{X}$, regarded as a reset map, and a set $J \subseteq X$, regarded as a forced transition set.

Definition 7 (see [10], run of a hybrid differential inclusion). A run of a hybrid differential inclusion $H=(X, F, R, J)$ is a pair $(\tau, x)$, consisting of a hybrid time trajectory $\tau$ and a map $x: \tau \rightarrow X$, that satisfies:

(1) discrete evolution: for all $i, x\left(\tau_{i+1}\right) \in R\left(x\left(\tau_{i}^{\prime}\right)\right)$;

(2) continuous evolution: if $\tau_{i}<\tau_{i}^{\prime}, x(\cdot)$ is a solution to the differential inclusion $\dot{x}(t) \in F(x)$ over the interval $\left[\tau_{i}, \tau_{i}^{\prime}\right]$ starting at $x\left(\tau_{i}\right)$, with $x(t) \notin J$ for all $t \in\left[\tau_{i}, \tau_{i}^{\prime}\right)$.

We use $\mathscr{R}_{H}\left(x_{0}\right)$ to denote the set of all runs of a hybrid differential inclusion $H=(X, F, R, J)$ starting at a state $x\left(\tau_{0}\right)=x_{0} \in X$.

Definition 8 (see [10]). Let $H=(X, F, R, J)$ be a hybrid differential inclusion. A set $K \subseteq X$ is called viable under a hybrid differential inclusion $H$, if for all $x_{0} \in K$, there exists an infinite run $(\tau, x) \in \mathscr{R}_{H}^{\infty}\left(x_{0}\right)$ viable in $K . K$ is called invariant under the hybrid differential inclusion $H$, if for all $x_{0} \in K$, all runs $(\tau, x) \in \mathscr{R}_{H}\left(x_{0}\right)$ are viable in $K$.

Proposition 9 (see [8]). The closed set $W \subset \mathbb{R}^{n}$ is said to be viable under the system (1), if and only if for any $x \in W$, the following formula is satisfied:

$$
\left(\bigcup_{u \in U} f(x, u)\right) \bigcap T_{K}(x) \neq \emptyset .
$$

For any interior point $x$ in the set $W$, the tangent cone $T_{K}(x)=$ $\mathbb{R}^{n}$, so the above formula is satisfied. Hence, if we want to judge the above formula, we should only consider the boundary point.

\section{The Viability of a Hybrid System}

To discuss the problem in $\mathbb{R}^{n}$, we assume $X=\mathbb{R}^{n}$ in the following paper.

Consider the following hybrid system $H=(X, F, R, J)$, and

$$
\dot{x} \in F(x)=f(x)+g(x) u, \quad x \in X, u \in U,
$$

where $f: \mathbb{R}^{n} \rightarrow \mathbb{R}^{n}$ and $g: \mathbb{R}^{n} \rightarrow \mathbb{R}^{m+n}$ are both Lipschitz functions. $U \subset \mathbb{R}^{m}$ is a convex set; it denotes

$$
U=\left\{u \in \mathbb{R}^{m} \mid h_{i}(u) \leq 0, i=1,2, \ldots, p\right\},
$$

where $h_{i}(u)(i=1,2, \ldots, p)$ are convex functions on the $\mathbb{R}^{n}$. $R$ is a reset map, and $J$ is a forced transition set.

Consider the following region $K$ :

$$
K=\left\{x \in \mathbb{R}^{n} \mid \varphi_{j}(x) \leq 0, j=1,2, \ldots, q\right\},
$$

and $\varphi_{j}(x)(j=1,2, \ldots, q)$ are sub-differentiable functions on $\mathbb{R}^{n}$. Furthermore, we assume that sub-differential $\partial \varphi_{j}(x)$ is a convex hull of many finite points. 
For hybrid time set $\tau=\left\{I_{i}\right\}_{i=0}^{N}$, where $\tau$ is interval sequence. For $i<N$, it has $I_{i}=\left[\tau_{i}, \tau_{i}^{\prime}\right]$, for all $i, \tau_{i} \leq \tau_{i}^{\prime}=\tau_{i+1}$. $x\left(\tau_{i}^{\prime}\right)$ are the points at which discrete transitions take place, $x\left(\tau_{i+1}\right)$ are the points after discrete transitions take place; that is,

$$
x\left(\tau_{i+1}\right)=R\left(x\left(\tau_{i}^{\prime}\right)\right), \quad i<N .
$$

On the other hand, we assume that the discrete transition does not occur infinite times within the limited time. The set $J$ is a forced transition set; that is, the discrete transition must happen for every point in $J$. Without generality, we assume that the set $K$ contains the forced transition set $J$ and the set $J$ contains countable transition points. For discussing easily, we still denote by $x\left(\tau_{i}^{\prime}\right)(i=0,1,2, \ldots, N-1)$. In addition, in order to describe the uncertainy in the hybrid differential system and to determine whether discrete transition will happen for every point $x$ in the set

$$
R^{-1}(X)=\{x \in X \mid R(x) \neq \emptyset\},
$$

we assume that

$$
R^{-1}(X) \subset K, \quad J \subset R^{-1}(X) ;
$$

it can prevent the system from death cycle. Obviously, the points which are in $R^{-1}(X) \backslash J$ may not be jump.

Let

$$
\varphi(x)=\max _{1 \leq j \leq q} \varphi_{j}(x) .
$$

Since the point $x \in \mathbb{R}^{n}$ satisfies

$$
\max _{1 \leq j \leq q} \varphi_{j}(x) \leq 0,
$$

which is equivalent to

$$
\varphi_{j}(x) \leq 0, \quad(j=1,2, \ldots, q),
$$

so the set $K$ can be denoted by the following formula:

$$
K=\left\{x \in \mathbb{R}^{n} \mid \varphi(x) \leq 0\right\} .
$$

Because $\varphi_{j}(x)(j=1,2, \ldots, m)$ are sub-differentiable, so $\varphi(x)$ is also sub-differentiable; since $\partial \varphi_{j}(x)$ is a convex hull of many finite points, the sub-differential of $\varphi(x)$ is also a convex hull of many finite points, marking

$$
\partial \varphi(x)=\operatorname{co}\left\{v^{1}, \ldots, v^{r}\right\}, \quad v^{i} \in \mathbb{R}^{n}, i=1,2, \ldots, r .
$$

Define matrix $B=\left(v^{1}, \ldots, v^{r}\right)^{T}$.

In nonsmooth optimization, two frequently used constraint qualifications:

constraint qualification 1 [8]: there exists $y^{0} \in \mathbb{R}^{n}$, such that $\varphi^{\prime}\left(x ; y^{0}\right)<0$;

constraint qualification 2 [21]: $\mathrm{cl} \gamma(x)=\Gamma(x)$, where

$$
\begin{aligned}
& \gamma(x)=\left\{y \in \mathbb{R}^{n} \mid \varphi^{\prime}(x ; y)<0\right\}, \\
& \Gamma(x)=\left\{y \in \mathbb{R}^{n} \mid \varphi^{\prime}(x ; y) \leq 0\right\} .
\end{aligned}
$$

Lemma 10 (see [14, 21]). If the set $K$ satisfied constraint qualification 1 or constraint qualification 2 at $x \in \mathbb{R}^{n}$, then $T_{K}(x)=\Gamma(x)$.

According to [20], we get the following Proposition 11 immediately.

Proposition 11 (see [20]). Assume that constraint qualification 1 or 2 is satisfied; then $T_{K}(x)=\left\{y \in \mathbb{R}^{n} \mid B y \leq 0\right\}$, where $B=\left(v^{1}, \ldots, v^{r}\right)^{T}, v^{i} \in \mathbb{R}^{n}(i=1,2, \ldots, r), \partial g(x)=$ $\operatorname{co}\left\{v^{1}, \ldots, v^{r}\right\}$.

Lemma 12 (see [10]). Consider a hybrid system $H=$ $(X, F, R, J)$ such that $F$ is Marchaud, $R$ is upper semicontinuous with closed domain, and $J$ is a closed set. A closed set $K \subseteq X$ is viable under $H$ if and only if

(1) $K \cap J \subseteq R^{-1}(K)$;

(2) $F(x) \cap T_{K}(x) \neq \emptyset, \forall x \in K \backslash R^{-1}(K)$.

Before we state Theorem 13, we construct the following inequality system:

$$
h_{i}(u) \leq 0, \quad i=1,2, \ldots, p, \quad B f(x)+B g(x) u \leq 0,
$$

where $u \in \mathbb{R}^{m}$ is a variable.

According to [20] and Lemma 12, we get the following theorem immediately.

Theorem 13. For the above hybrid system $H=(X, F, R$, J), if

$$
\begin{aligned}
K & =\left\{x \in X \mid \varphi_{j}(x) \leq 0, j=1,2, \ldots, m\right\} \\
& =\{x \in X \mid \varphi(x) \leq 0\}
\end{aligned}
$$

satisfies constraint qualification 1 or 2 , then the set $K$ is viable under the hybrid system $\mathrm{H}$ if and only if

(1) discrete transition (or jump) must take place: $\varphi\left(x\left(\tau_{i+1}\right)\right) \leq 0, i=0,1,2, \ldots, N-1$.

(2) continuous section: for each fixed point $x \in K \backslash R^{-1}(K)$ inequality system (18) is solvable.

Proof. Under the above assumptions, it is sufficient to show that Theorem 13(1) is equivalent to Lemma 12(1) and Theorem 13(2) is equivalent to Lemma 12(2).

In Lemma 12(1), $K \cap J \subseteq R^{-1}(K)$ is equivalent to the following statement: when discrete transition (or jump) must happen $(x \in K \cap J)$ for every $x \in K$, then the point after the transition (or jump) must be in the set $K(R(x) \cap$ $K \neq \emptyset)$. Based on the aforementioned assumptions, for the jump point $x\left(\tau_{0}^{\prime}\right), x\left(\tau_{1}^{\prime}\right), \ldots, x\left(\tau_{N-1}^{\prime}\right)$ contained in the set $J$, we only need to show that the point will still be in $K$ after the jump $\left(x\left(\tau_{i+1}\right)=R\left(x\left(\tau_{i}^{\prime}\right)\right),(i=0,1,2, \ldots, N-1)\right.$. That is, $x\left(\tau_{i+1}\right) \in K(i=0,1,2, \ldots, N-1)$. Since $K=\{x \in X \mid$ $\varphi(x) \leq 0\}, \varphi\left(x\left(\tau_{i+1}\right)\right) \leq 0(i=0,1,2, \ldots, N-1)$. Hence Theorem 13(1) is equivalent to Lemma 12(1).

The Lemma 12(2) is sufficient to show that the changes is possible $\left(F(x) \cap T_{K}(x) \neq \emptyset\right)$ for continuous section in $K$, 
when discrete transition point (or jump point) $(R(x) \cap K=\emptyset)$ will be not in $K$ after the jump. The set $K$ satisfies constraint qualification 1 or 2 ; then $T_{K}(x)=\left\{y \in \mathbb{R}^{n} \mid B y \leq 0\right\}$. We set $f(x, u)=f(x)+g(x) u$ in Proposition 9; then the set $K$ is viable under the hybrid system $H$ if and only if the following formula is satisfied:

$$
\left(\bigcup_{u \in U}(f(x)+g(x) u)\right) \bigcap T_{K}(x) \neq \emptyset
$$

where $x$ is a fixed point in $K \backslash R^{-1}(K)$. Consider the expressions of the set $U$ and $T_{K}(x)$; the above expression is equivalent to

$$
\begin{aligned}
& \left\{f(x)+g(x) u \mid h_{i}(u) \leq 0\right. \\
& i=1,2, \ldots, p\} \bigcap\left\{y \in \mathbb{R}^{n} \mid B y \leq 0\right\} \neq \emptyset
\end{aligned}
$$

Obviously, the above equation is equivalent to following solvable system:

$$
\begin{gathered}
h_{i}(u) \leq 0, \quad i=1,2, \ldots, p, \quad B y \leq 0, \\
y=f(x)+g(x) u .
\end{gathered}
$$

In (22), we set that $y=f(x)+g(x) u$ substitute into $B y \leq$ 0 ; then we can obtain (18). Also, we can obtain (22) by substituting $y=f(x)+g(x) u$ into (18). This shows that the system (18) is equivalent to the system (22). This completes the proof.

\section{The Viability of a Hybrid Differential Inclusion}

Hybrid differential inclusion can describe a hybrid system in a wide range of significance.

Consider the following hybrid differential system $H=$ $(X, F, R, J)$, and

$$
\dot{x} \in F(x)=\operatorname{co}\left\{f_{i}(x) \mid i=1,2, \ldots, p\right\}, \quad x \in X,
$$

where $f_{i}(x)(i=1,2, \ldots, p)$ are functions on $X$. $R$ is a reset map, and $J$ is a forced transition set.

Consider the following region $K$ :

$$
K=\left\{x \in X \mid g_{j}(x) \leq 0, j=1,2, \ldots, m\right\},
$$

where $g_{j}(x)(j=1,2, \ldots, m)$ are sub-differentiable functions on $X$. We further assume that the functions $g_{j}(x)$ are subdifferentiable, and sub-differential $\partial g_{j}(x)$ is a convex hull with finite point set. Let

$$
g(x)=\max _{1 \leq j \leq m} g_{j}(x) ;
$$

then the set $K$ can be rewritten as

$$
K=\{x \in X \mid g(x) \leq 0\} .
$$

Since $g_{j}(x)(j=1,2, \ldots, m)$ are sub-differentiable, $g(x)$ is sub-differentiable. Because $\partial g_{j}(x)$ is a convex hull with finite point set, sub-differential of $g(x)$ is also a convex hull with finite point set, denoted by

$$
\partial g(x)=\operatorname{co}\left\{v^{1}, \ldots, v^{q}\right\}, \quad v^{i} \in X \quad(i=1,2, \ldots, q) .
$$

Theorem 14. For the above hybrid differential inclusion $H=$ $(X, F, R, J)$, if

$$
\begin{aligned}
K & =\left\{x \in X \mid g_{j}(x) \leq 0, j=1,2, \ldots, m\right\} \\
& =\{x \in X \mid g(x) \leq 0\}
\end{aligned}
$$

satisfies constraint qualification 1 or 2 , then the set $K$ is viable under the hybrid differential inclusion $H$ if and only if

(1) discrete transition (or jump) must take place: $g\left(x\left(\tau_{i+1}\right)\right) \leq 0, i=0,1,2, \ldots, N-1 ;$

(2) continuous section: Optimal value of the following linear programming problem $(P)$ is zero for each $x \in$ $K \backslash R^{-1}(K)$. Consider

$$
\begin{aligned}
\min & \omega \\
\text { s.t. } & \sum_{i=1}^{p} \lambda_{i} B f_{i}\left(x_{i}\right)+(\omega, \ldots, \omega)^{T} \leq 0, \\
& \sum_{i=1}^{p} \lambda_{i}=1, \\
& \lambda_{i} \geq 0, \quad i=1,2, \ldots, p, \\
& \omega \geq 0, \\
\text { where } B= & \left(v^{1}, \ldots, v^{q}\right)^{T} .
\end{aligned}
$$

Proof. Under the above assumptions, it is sufficient to show that Theorem 14(1) is equivalent to Lemma 12(1) and Theorem 14(2) is equivalent to Lemma 12(2).

In Lemma 12(1), $K \cap J \subseteq R^{-1}(K)$ is equivalent to the following statement: when discrete transition (or jump) must happen $(x \in K \cap J)$ for every $x \in K$, then the point after the transition (or jump) must be in the set $K(R(x) \cap K \neq \emptyset)$. Based on the aforementioned assumptions, for the jump point $x\left(\tau_{0}^{\prime}\right), x\left(\tau_{1}^{\prime}\right), \ldots, x\left(\tau_{N-1}^{\prime}\right)$ in the set $J$, we only need to show that the point after the jump $\left(\left(x\left(\tau_{i+1}\right)=R\left(x\left(\tau_{i}^{\prime}\right)\right), i=\right.\right.$ $0,1,2, \ldots, N-1))$ will be still in $K$. That is, $x\left(\tau_{i+1}\right) \in K$ $(i=0,1,2, \ldots, N-1)$. Since $K=\{x \in X \mid g(x) \leq 0\}$, $g\left(x\left(\tau_{i+1}\right)\right) \leq 0(i=0,1,2, \ldots, N-1)$. Hence Theorem 14(1) is equivalent to Lemma $12(1)$.

In Lemma 12(2), we noticed that when discrete transition point (or jump point) after the jump $(R(x) \cap K=\emptyset)$ will be not in $K$, then the changes are possible $\left(F(x) \cap T_{K}(x) \neq \emptyset\right)$ for continuous section in $K$. Since the set $K$ satisfies constraint qualification 1 or 2 ,

$$
\begin{gathered}
T_{K}(x)=\{y \in X \mid B y \leq 0\}, \quad B=\left(v^{1}, \ldots, v^{q}\right)^{T}, \\
v^{i} \in X \quad(i=1,2, \ldots, q), \quad \partial g(x)=\operatorname{co}\left\{v^{1}, \ldots, v^{q}\right\} .
\end{gathered}
$$


In addition,

$$
F(x)=\operatorname{co}\left\{f_{i}(x) \mid i=1,2, \ldots, p\right\} ;
$$

then the condition

$$
F(x) \cap T_{K}(x) \neq \emptyset
$$

and the following problem which has a solution

$$
\begin{aligned}
& B\left(\sum_{i=1}^{p} \lambda_{i} f_{i}(x)\right)=\sum_{i=1}^{p} \lambda_{i} B f_{i}(x) \leq 0, \\
& \sum_{i=1}^{p} \lambda_{i}=1, \quad \lambda_{i} \geq 0, \quad i=1,2, \ldots, p,
\end{aligned}
$$

are equivalent, and also are equivalent to the linear programming problem $(\mathrm{P})$ in which the optimal solution is zero. This completes the proof.

Lemma 15 (see [10]). Let hybrid differential inclusion be $H=$ $(X, F, R, J)$ such that $F$ is Marchaud and Lipschitz, and $J$ is a closed set. A closed set $K \subseteq X$ is invariant under $H$ if and only if

(1) $R(K) \subseteq K$;

(2) $F(x) \subseteq T_{K}(x)$, for all $x \in K \backslash J$.

Theorem 16. $H=(X, F, R, J)$ is a hybrid differential inclusion as above; if the set $K$ satisfies constraint qualification 1 or 2 , then the set $K=\{x \in X \mid g(x) \leq 0\}$ is invariant under hybrid differential inclusion $H$ if and only if

(1) discrete transition (or jump) must take place: $g\left(x\left(\tau_{i+1}\right)\right) \leq 0, i=0,1,2, \ldots, N-1$; uncertainty section: $g(R(x)) \leq 0$, for all $x \in K \backslash J$;

(2) continuous section: $B f_{i}(x) \leq 0, i=1,2, \ldots, p$, for all $x \in K \backslash J$.

Proof. Under the above assumptions, it is sufficient to show that Theorem 16(1) is equivalent to Lemma 15(1) and Theorem 16(2) is equivalent to Lemma 15(2).

In Lemma 15(1), to verify $R(K) \subseteq K$, we just need to show that the point after the transition (or jump) must be in the set $K(R(K) \subseteq K)$, when discrete transition (or jump) must happen $(x \in J)$ for every $x \in K$. By the previous assumptions, the jump point contained in the set $J$ should show that the point will be still in $K$ after the jump. That is, there exists $x\left(\tau_{i+1}\right) \in R\left(x\left(\tau_{i}^{\prime}\right)\right)(i=0,1,2, \ldots, N-1)$, such that $x\left(\tau_{i+1}\right) \in K(i=0,1,2, \ldots, N-1)$; that is, $g\left(x\left(\tau_{i+1}\right)\right) \leq 0$ $(i=0,1,2, \ldots, N-1)$. In addition, for each $x$ in $K \backslash J$, the point after the transition (or jump) will still be in $K$; that is, $g(R(x)) \leq 0, x \in K \backslash J$. Hence, Theorem 16(1) is equivalent to Lemma 15(1).

In Lemma 15(2), for $x \in K \backslash J, F(x) \subseteq T_{K}(x)$ is equivalent to the following statement: if the continuous evolution is possible $(x \notin J)$, then all solutions of $\dot{x} \in F(x)$ are all in $K\left(F(x) \subseteq T_{K}(x)\right)$. The set $K$ satisfies constraint qualification 1 or 2 ; then

$$
T_{K}(x)=\{y \in X \mid B y \leq 0\} .
$$

Moreover,

$$
F(x)=\operatorname{co}\left\{f_{i}(x) \mid i=1,2, \ldots, p\right\}
$$

then the condition $F(x) \subseteq T_{K}(x)$ is equivalent to $B f_{i}(x) \leq 0$ $(i=1,2, \ldots, p)$, which completes the proof of Theorem 16 .

\section{Example}

We provide here an example that better illustrates the class of hybrid systems where our theoretical framework is relevant.

Consider the differential inclusion $H=(X, F, R, J)$, where

$$
\begin{gathered}
F(x)=\operatorname{co}\left\{f_{1}(x), f_{2}(x)\right\}, \quad x \in \mathbb{R}^{2}, \\
f_{1}(x)=\left(x_{1}+x_{2}, x_{2}+1\right)^{T}, \quad f_{2}(x)=\left(x_{1}+x_{2}+1, x_{2}\right)^{T}, \\
J=\left\{x \in \mathbb{R}^{2} \mid x_{1} \geq 0, x_{2} \geq 0, x_{1}+x_{2}-1 \leq 0\right\}, \\
K=\left\{x \in \mathbb{R}^{2} \mid g(x) \leq 0\right\}, \\
g(x)=\max \left\{-x_{1},-x_{2}, x_{1}^{2}+x_{2}^{2}-1\right\}, \\
R(x)=\left(x_{1}+\frac{1}{2}, x_{2}-\frac{1}{3}\right)^{T}, \quad x=\left(x_{1}, x_{2}\right)^{T} .
\end{gathered}
$$

We can easily conclude that $g(x)$ is a sub-differentiable function, and the set $K$ is a quarter of the unit circle.

(1) Viability discrimination of the point $x^{(1)}=(0,1)^{T}$ under the hybrid differential inclusion $H$ : by the definition and operation of the sub-differential,

$$
\partial g\left(x^{(1)}\right)=\operatorname{co}\left\{(-1,0)^{T},(0,2)^{T}\right\} .
$$

Obviously, the point $x^{(1)}$ is in the set $J$, so discrete transition (or jump) must take place. The point after jump is $R\left((0,1)^{T}\right)=(1 / 2,2 / 3)^{T}$. It can be shown that the point $(1 / 2,2 / 3)^{T}$ in $K \backslash J$ and it is the interior point of the set $K \backslash J$. Hence the point $x^{(1)}=(0,1)^{T}$ in $K$ satisfied the viability condition.

(2) Viability discrimination of the point $x^{(2)}=$ $(1 / 2, \sqrt{3} / 2)^{T}$ under the hybrid differential inclusion $H$ : Obviously, the point $x^{(2)}=(1 / 2, \sqrt{3} / 2)^{T}$ is in the set $K \backslash J$ and it is the boundary point. Viability discrimination of the point $x^{(2)}=(1 / 2, \sqrt{3} / 2)^{T}$ 
is equivalent to the following linear programming problem (37) in which the optimal solution is zero

$$
\begin{array}{ll}
\min & \omega \\
\text { s.t. } & \sum_{i=1}^{p} \lambda_{i} B f_{i}(x)+(\omega, \ldots, \omega)^{T} \leq 0, \\
& \lambda_{i} \geq 0, \quad i=1,2 \\
& \lambda_{1}+\lambda_{2}=1 \\
& \omega \geq 0
\end{array}
$$

We can obtain sub-differential $\partial g\left(x^{(2)}\right)=(1, \sqrt{3})^{T}$; hence, $B=(1, \sqrt{3})$,

$$
\begin{gathered}
f_{1}\left(x^{(2)}\right)=\left(\frac{1+\sqrt{3}}{2}, \frac{2+\sqrt{3}}{2}\right)^{T}, \\
f_{2}\left(x^{(2)}\right)=\left(\frac{3+\sqrt{3}}{2}, \sqrt{3}\right)^{T} .
\end{gathered}
$$

Consequently, the linear programming problem reduces to

$$
\begin{array}{ll}
\min & \omega \\
\text { s.t. } & \frac{4+3 \sqrt{3}}{2} \lambda_{1}+\frac{9+\sqrt{3}}{2} \lambda_{2}-\omega \leq 0, \\
& \lambda_{i} \geq 0, \quad i=1,2 . \\
& \lambda_{1}+\lambda_{2}=1, \\
& \omega \geq 0 .
\end{array}
$$

Obviously, $\omega=0$ is not the optimal value of constrained optimization. Hence $x^{(2)}=(1 / 2, \sqrt{3} / 2)^{T}$ does not satisfy the conditions for the viability of differential inclusion.

\section{Conflict of Interests}

The authors declare that there is no conflict of interests regarding the publication of this paper.

\section{References}

[1] M. S. Shaikh and P. E. Caines, "On the hybrid optimal control problem: theory and algorithms," IEEE Transactions on Automatic Control, vol. 52, no. 9, pp. 1587-1603, 2007.

[2] M. Boccadoro, Y. Wardi, M. Egerstedt, and E. Verriest, "Optimal control of switching surfaces in hybrid dynamical systems," Journal of Discrete Event Dynamic Systems, vol. 15, no. 4, pp. 433-448, 2005.

[3] D. Liberzon, Calculus of Variations and Optimal Control Theory, A Concise Introduction, Princeton University Press, Princeton, NJ, USA, 2012.

[4] H. S. Witsenhausen, "A class of hybrid-state continuous-time dynamic systems," IEEE Transactions on Automatic Control, vol. 11, pp. 161-167, 1966.
[5] H. Wong-Toi, "Synthesis of controllers for linear hybrid automata," in Proceedings of the 36th IEEE Conference on Decision and Control, pp. 4607-4613, San Diego, Calif, USA, December 1997.

[6] J. Lygeros, C. Tomlin, and S. Sastry, "Controllers for reachability specifications for hybrid systems," Automatica, vol. 35, no. 3, pp. 349-370, 1999.

[7] C. J. Tomlin, J. Lygeros, and S. Shankar Sastry, "A game theoretic approach to controller design for hybrid systems," Proceedings of the IEEE, vol. 88, no. 7, pp. 949-969, 2000.

[8] J.-P. Aubin, Viability Theory, Birkhäuser, Boston, MA, 1991.

[9] J.-P. Aubin, "A survey of viability theory," SIAM Journal on Control and Optimization, vol. 28, no. 4, pp. 749-788, 1990.

[10] J.-P. Aubin, J. Lygeros, M. Quincampoix, S. Sastry, and N. Seube, "Impulse differential inclusions: a viability approach to hybrid systems," IEEE Transactions on Automatic Control, vol. 47, no. 1, pp. 2-20, 2002.

[11] P. Cardaliaguet, M. Quincampoix, and P. Saint-Pierre, "Pursuit differential games with state constraints," SIAM Journal on Control and Optimization, vol. 39, no. 5, pp. 1615-1632, 2001.

[12] Y. Gao, J. Lygeros, and M. Quincampoix, "On the reachability problem for uncertain hybrid systems," IEEE Transactions on Automatic Control, vol. 52, no. 9, pp. 1572-1586, 2007.

[13] Y. Gao, J. Lygeros, M. Quincampoix, and N. Seube, "On the control of uncertain impulsive systems: approximate stabilization and controlled invariance," International Journal of Control, vol. 77, no. 16, pp. 1393-1407, 2004.

[14] M. Quincampoix and N. Seube, "Stabilization of uncertain control systems through piecewise constant feedback," Journal of Mathematical Analysis and Applications, vol. 218, no. 1, pp. 240-255, 1998.

[15] F. Blanchini, "Set invariance in control," Automatica, vol. 35, no. 11, pp. 1747-1767, 1999.

[16] B. E. A. Milani and C. E. T. Dórea, "On invariant polyhedra of continuous-time systems subject to additive disturbances," Automatica, vol. 32, no. 5, pp. 785-789, 1996.

[17] J.-P. Aubin, Optima and Equilibria: An Introduction to Nonlinear Analysis, vol. 140, Springer, Berlin, Germany, 1993.

[18] Y. Gao, "Determining the viability for a affine nonlinear control system," Control Theory and Applications, vol. 26, no. 6, pp. 654656, 2009.

[19] N. U. Ahmed, "Optimal relaxed controls for systems governed by impulsive differential inclusions," Nonlinear Functional Analysis and Applications, vol. 10, no. 3, pp. 427-460, 2005.

[20] Y. Gao, "Viability criteria for differential inclusions," Journal of Systems Science \& Complexity, vol. 24, no. 5, pp. 825-834, 2011.

[21] Y. Gao, "Determining the viability for a class of nonlinear control systems," Information and Control, vol. 34, no. 4, pp. 510-512, 2005. 


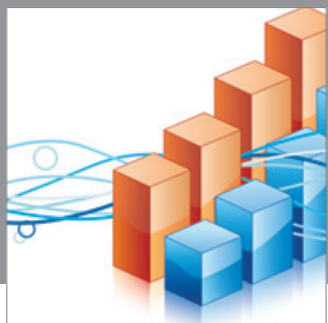

Advances in

Operations Research

mansans

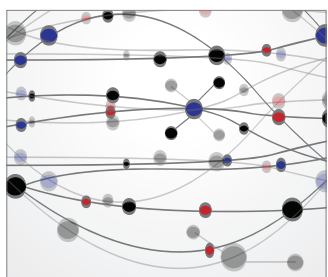

The Scientific World Journal
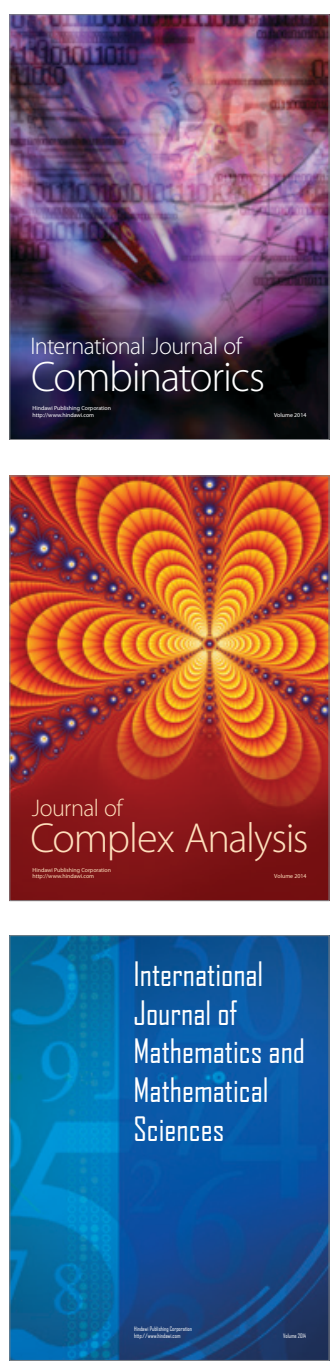
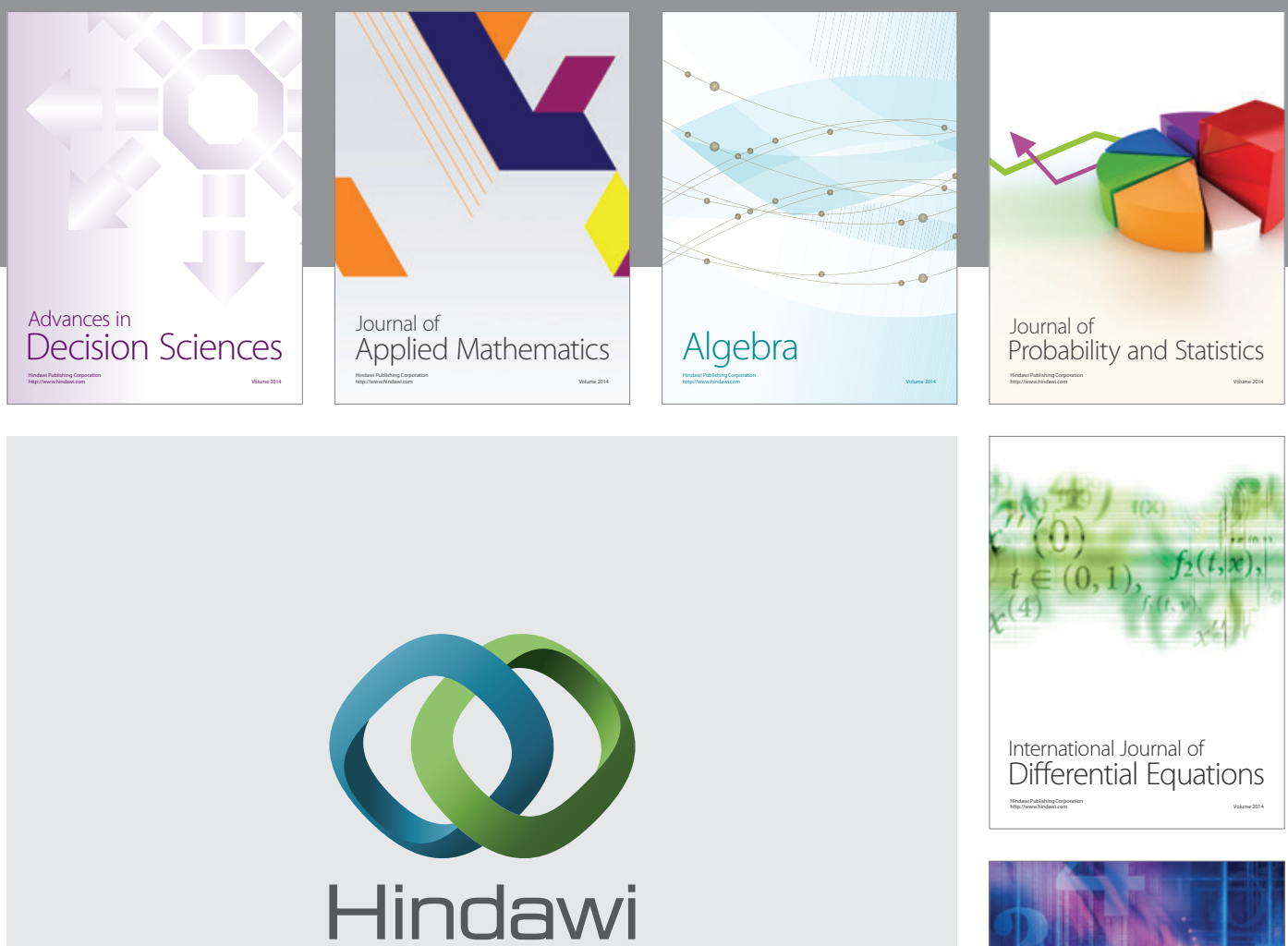

Submit your manuscripts at http://www.hindawi.com
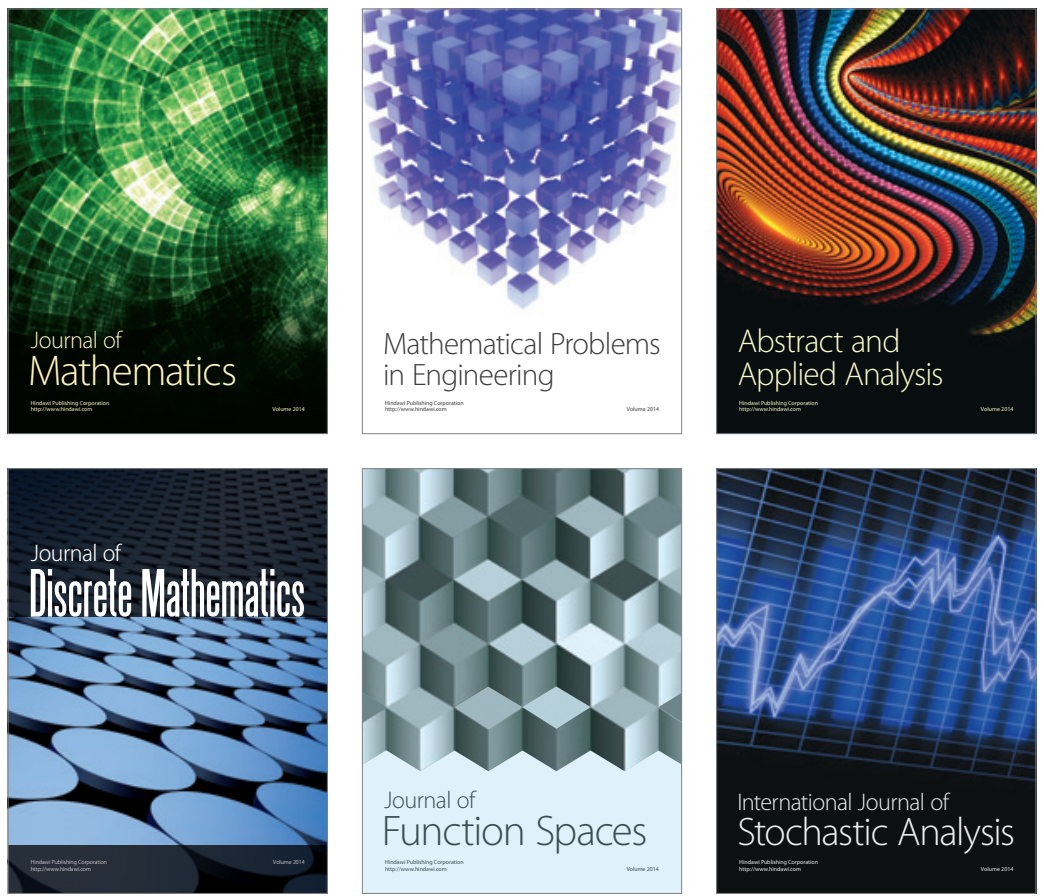

Journal of

Function Spaces

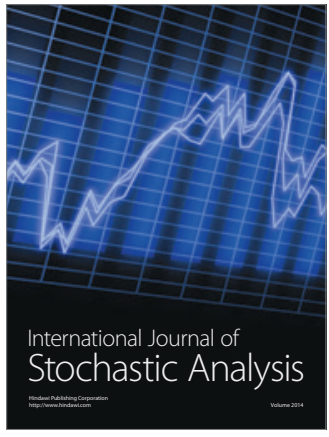

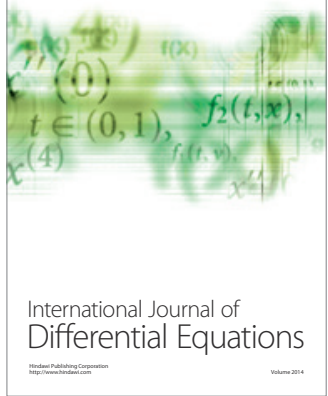
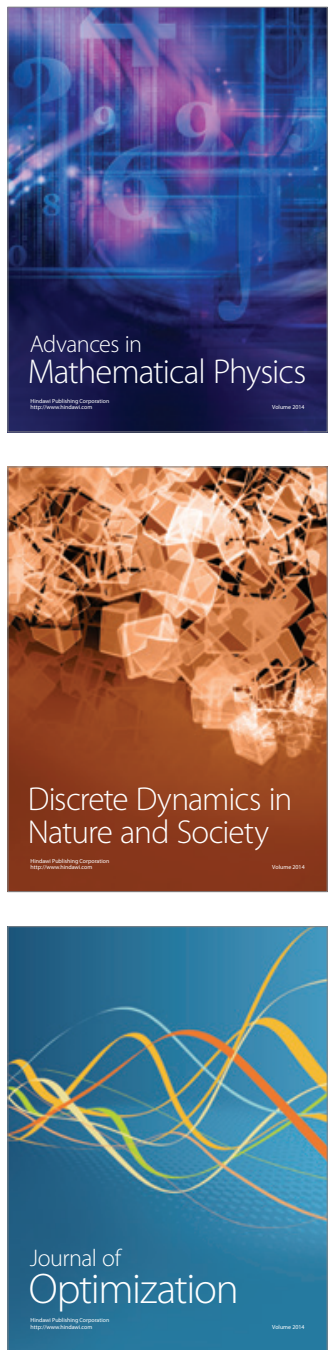